\title{
High Resolution IgH Repertoire Analysis Reveals the Human Fetal Liver as the Origin of Life-long, Innate B Lymphopoiesis
}

Anindita Roy ${ }^{1 *}$, Vojtech Bystry ${ }^{2 *}$, Georg Bohn ${ }^{3}$, Katerina Goudevenou ${ }^{3}$, Tomas Reigl $^{2}$, Maria Papaioannou ${ }^{3}$, Adam Krejci ${ }^{2,4}$, Sorcha O'Byrne ${ }^{1}$, Aristeidis Chaidos ${ }^{3}$, Andrea Grioni ${ }^{2,5}$, Nikos Darzentas ${ }^{2}$, Irene AG Roberts ${ }^{1,6^{*}}$, Anastasios Karadimitris $^{3^{*}}$

Introduction: Development of mature B cells depends upon the expression of a functional B cell receptor (BCR) and of its immunoglobulin (Ig) heavy (H) and light (L) chains. The molecular hallmark of B cell development, somatic recombination of the genes that encode the $\operatorname{IgH}(\mathrm{V}, \mathrm{D}$ and $\mathrm{J})$ and $\operatorname{IgL}(\mathrm{V}$ and $\mathrm{J})$ chains, takes place in B cells in primary B lymphopoiesis sites throughout development (i.e. fetal liver (FL), fetal bone marrow (FBM) and adult BM). However, the spatiotemporal relationship between the IgH repertoire in FL with that in FBM, and the impact of the fetal Ig repertoire on the long-term repertoire present in post-natal life, as well as the link between this and the development of disease, are largely unknown.

Aim: To gain insights into the ontogeny of the human innate B cell repertoire by performing high resolution analysis of the IgH-Cmu repertoire of human FL, FBM and post-natal B cells.

Methods: CD34negCD19pos B cells from human 2nd trimester FL and FBM, child peripheral blood (cPB) and adult peripheral blood (aPB) were isolated by flow sorting. For initial assessment of the IgM repertoire ontogeny in FL and FBM B cells, we performed CDR3 fragment length analysis of VH1-VH6 families in the B cells. To gain further insights into the ontogeny of IgH diversification, we employed 454 next generation sequencing (NGS)-based analysis of the IgVHCmu mRNA repertoire in FL, FBM and postnatal B cells. In total, 20 libraries generated from 17 individual, flow-sorted CD34negCD19pos B cell samples were sequenced: 5 FL (4 performed in independent duplicate libraries; GA 14-18 weeks), 3 FBM (GA 13-17 weeks), 3 cPB (age 4mo-4yrs) and 5 aPB (age 29-53 yrs) samples. Bioinformatic analysis was performed with the ARResT/Interrogate immunoprofiler, IMGT tools, and appropriate statistics.

Results: Spectratyping and NGS data showed that the molecular mechanisms responsible for VDJ recombination-dependent repertoire diversification, such as 
convergent recombination, are active in early B cell development and as efficient as postnatal B cells in both FL and FBM. Comparably diversified B cell lymphopoiesis exists contemporaneously in FL and FBM. However, analysis of the top 100 most abundant clonotypes in each developmental stage showed that their mean abundance in FBM B cells was 10 times lower (0.12\%) than in FL B cells $(1.2 \%$; $\mathrm{p}<0.0001)$. Reflecting this relative paucity of expanded clonotypes amongst FBM B cells, analysis of the 100 most abundant clonotypes across all developmental stages showed that 0 were present in FBM, compared to 65 in FL, 23 in $\mathrm{CPB}$, and $12 \mathrm{in} \mathrm{PBB}$. We found that this paucity of clonotypic expansions in FBM is likely due to the significant decrease in sIgM-expressing transitional and naïve B cells compared to FL (FL: $4.76 \pm 0.8 \%$ vs. FBM: $1.9 \pm 0.4 \%$, p $<0.01$; and FL: $2.8 \pm 0.9 \%$ vs. FBM: $0.7 \pm 0.2 \%, \mathrm{p}<0.05$ respectively). Strikingly, $0.4 \%$ of all fetal life clonotypes, comprising $18.7 \%$ of the whole repertoire, were shared with the PB IgM B cell repertoire in children and adults, a pattern that is particularly pronounced for the VH6-1 gene. Reflecting the presence of high abundance clonotypes in FL but not FBM, the mean abundance of the clonotypes shared between FL and postnatal B cells was 38-fold higher than that of FBM $(0.77 \% \mathrm{v}$ $0.02 \%, \mathrm{p}=0.001)$. Selection of these shared or public clonotypes is likely to occur in an HLA- and thus T cell-independent manner, and is therefore consistent with IgM innate humoral immune responses. Lastly, chronic lymphocytic leukemia (CLL) is the most common IgM+ mature B cell malignancy with subsets of patients with stereotyped BCR collectively accounting for $~ 30 \%$ of cases; we found stereotypic IgH receptors corresponding to 16/19 major CLL subsets in $3 / 5$ FL samples and in all FBM and postnatal samples.

Conclusions: The lack of mature, antigen-responsive B cells in FBM and the pronounced clonotypic expansions in FL suggest that FL is the main source of IgM natural immunity during the 2nd trimester. Our results are consistent with persisting fetal IgM+ B cells being an important source of the natural IgM repertoire also in adult life. Further, the origins of specific stereotypic IgM+ BCR associated with CLL, can be traced back to fetal B cell lymphopoiesis, suggesting that persisting fetal B cells can be subject to malignant transformation late in life. Overall, these novel data provide unique insights into the ontogeny of physiological and malignant B lymphopoiesis that spans the human lifetime. 